\title{
Concurrent training effects on cardiac muscle structure and function of middle-aged obese men.
}

Keryma C. S. Mateus (IC), Arthur F. Gáspari (PG), Amanda V. Sardeli (PG), Guilherme De Rossi (PG), Wilson Nadruz Jr. (PQ), Mara T. Chacon-Mikahil (PQ), Cláudia R. Cavaglieri (PQ).

\begin{abstract}
As obese subjects have a higher risk to develop abnormal changes in the morphology and function of the heart, this study aimed to assess whether a Combined or Concurrent Training protocol (CT) - strength training plus aerobic training performed in the same session -, lasting 24 weeks, would cause beneficial structural and functional adaptations in the heart of obese middle-aged men. To this end, we use conventional echocardiography and Doppler analysis pre and post the of training or control period.
\end{abstract}

Key words: obesity, echocardiographic variables, concurrent training.

\section{Introduction}

Currently, obesity is a major public health problem worldwide. This condition is capable to produce functional and morphological changes in the cardiovascular system, which can lead to cardiovascular diseases. Although, physical training has also numerous effects on the morphological and functional variables of the human heart, there are few studies showing the effect of CT on these variables in obese. This training is recommended by international organizations for maintenance and/or improvement of health. Thus, the aim of this study was to investigate the effect of a 24-week CT protocol on morphological and functional cardiac variables of middle-age men with obesity grade I.

\section{Results and Discussion}

Twenty-eight middle-aged men (24.9> BMI $\left.<30 \mathrm{~kg} \cdot \mathrm{m}^{-2}\right)$ divided into control group $(\mathrm{CG}=12)$ and Combined Training Group (GTC = 16) participate in the study. The CT program consisted of strength exercises (6 exercises / 3 sets / 6-10 repetitions / 60-90s rest between sets) and aerobic exercise (30min of walking or running at $50-85 \%$ of $\mathrm{VO}_{2 \text { peak }}$ ) in the same session, $3 /$ week for 24 weeks. The $\mathrm{VO}_{2 \text { peak }}$ and muscle strength were measured by $\mathrm{VO}_{2 \max }$ test and test of one repetition maximum (1RM), respectively. The evaluation of the functional and morphologic cardiac variables was performed by echocardiography and Doppler analysis. All volunteers kept the eating pattern during the study. Statistical Analysis: Data were compared by two-way ANOVA followed by post hoc Tukey ( $p$ $\leq 0.05)$. Data are presented in $\Delta \%$ and standard deviation. Results: There was significant improvement in muscle strength and $\mathrm{VO}_{2 \text { peak. }}$ Diastolic function variable (E/A) and the systolic function variable $(\mathrm{Sm})$ had interaction group ${ }^{\star}$ time, however the post hoc showed no differences.

Table 1. Morphological and functional cardiac parameters of Control Group (CG) and Combined Training Group (CT).

\begin{tabular}{|l|c|c|}
\hline Functional variables & CG $\Delta \%$ & CTG $\Delta \%$ \\
\hline Muscle Strength & $0.93 \pm 7.4$ & $22.85 \pm 12.84^{*}$ \\
\hline VO ${ }_{\text {peak }}$ & $2.21 \pm 6.65$ & $10.43 \pm 8.28^{*}$ \\
\hline Heart variables & & \\
\hline Aortic diameter & $1.18 \pm 6$ & $-0.69 \pm 6.25$ \\
\hline Left atrial diameter & $3.31 \pm 10.71$ & $0.13 \pm 7.69$ \\
\hline $\begin{array}{l}\text { LV end-diastolic } \\
\text { volume }\end{array}$ & $-1.75 \pm 5.35$ & $1.26 \pm 4.81$ \\
\hline $\begin{array}{l}\text { LV end-systolic } \\
\text { volume }\end{array}$ & $-1.78 \pm 8.26$ & $2.27 \pm 7.85$ \\
\hline RWT & $7.50 \pm 11.21$ & $3.85 \pm 15.22$ \\
\hline Stroke volume & $-4.58 \pm 13.16$ & $1.36 \pm 15.91$ \\
\hline LV mass index & $5.25 \pm 15.72$ & $7.32 \pm 16.76$ \\
\hline Ejection Fraction & $2.01 \pm 10.10$ & $-0.93 \pm 8.30$ \\
\hline Sm & $-5.50 \pm 14.07$ & $12.14 \pm 24.44^{\#}$ \\
\hline E/A & $-16.42 \pm 15.60$ & $24.11 \pm 38.65^{\#}$ \\
\hline E/Em & $11.76 \pm 20.34$ & $1.42 \pm 21.88$ \\
\hline Em/Am & $-6.16 \pm 25.77$ & $21.33 \pm 35.01$ \\
\hline
\end{tabular}

LV-left ventricle; RWT- Relative wall thickness; \#-p valor group ${ }^{*}$ time (ANOVA); ${ }^{*}-p \leq 0.05$

\section{Conclusions}

The CT was not able to promote significant changes in the morphological and functional cardiac variables. However, it was effective in improving the functional capabilities.

\section{Acknowledgement}

Laboratory of Exercise Physiology - Unicamp and volunteers. Funding: FAPESP and CNPq. 